\title{
Production and purification of mannan oligosaccharide with epithelial tight junction enhancing activity
}

\author{
Chatchai Nopvichai ${ }^{1}$, Thanapon Charoenwongpaiboon ${ }^{1}$, Navaporn Luengluepunya ${ }^{1}$, Kazuo Ito ${ }^{2}$, Chatchai \\ Muanprasat $^{3}$, Rath Pichyangkura ${ }^{\text {Corresp. } 1}$ \\ 1 Department of Biochemistry, Faculty of Science, Chulalongkorn University, Bangkok, Thailand \\ ${ }^{2}$ Graduate School of Science, Osaka City University, Osaka, Japan \\ 3 Department of Physiology, Faculty of Science, Mahidol University, Bangkok, Thailand \\ Corresponding Author: Rath Pichyangkura \\ Email address: prath@chula.ac.th
}

Background. Mannanan oligosaccharide (MOS) is well-known as effective supplement food for livestock to increase their nutrients absorption and health status, but the structure and identification of bioactive MOS remain unclear. In this study, MOS production was accomplished, using enzymatic hydrolysis of pretreated coconut meal substrate with recombinant mannanase.

Methods. Mannanase gene was cloned from Bacillus subtilis CAE24, then expressed in BL21. Purified Mannanase exhibit stability over a wide range of $\mathrm{pH}$ and temperature from $\mathrm{pH} 6-8$ and $4{ }^{\circ}$ Celsius to 70 ${ }^{\circ}$ Celsius, respectively. SEM analysis revealed that sonication could change the surface characteristic of copra meal, which gave better MOS yield, compared to untreated substrates. The separation and purification of each MOS were achieved using Biogel-P2 column chromatography. Determination of biological active MOS species was also investigated. T84 cells were cultured and treated with each of the purified MOS species to determine their tight junction enhancing activity.

Results. Scanning electron microscope imaging showed that pretreatment using sonication could disrupt the surface of copra meal better than grinding alone, which can improve the production of MOS.

Pentamer of MOS (M5) significantly increased tight junction integration of T84 cells measured with TEER $(p<0.0001)$. 
1 Production and purification of mannan

2 oligosaccharide with epithelial tight junction

3 enhancing activity

4

5

6

7

8

9

Chatchai Nopvichai ${ }^{1}$, Thanapon Charoenwongpaiboon ${ }^{1}$, Navaporn Luengluepunya ${ }^{1}$, Kazuo Ito $^{2}$, Chatchai Muanprasat ${ }^{3}$, Rath Pichyangkura ${ }^{1}$

${ }^{1}$ Department of Biochemistry, Faculty of Science, Chulalongkorn University, Bangkok, Thailand.

${ }^{2}$ Graduate School of Science, Osaka City University, Osaka, Japan.

${ }^{3}$ Department of Physiology, Faculty of Science, Mahidol University, Bangkok, Thailand.

Corresponding Author:

Rath Pichyangkura ${ }^{1}$

Department of Biochemistry, Fl.5, Klum Watcharobol Bld.

Faculty of Science, Chulalongkorn University,

Payathai Rd., Bangkok 10330 Thailand

Email address: prath@chula.ac.th 
43 Abstract

44 Background. Mannanan oligosaccharide (MOS) is well-known as effective supplement food for livestock to increase their nutrients absorption and health status, but the structure and identification of bioactive MOS remain unclear. In this study, MOS production was accomplished, using enzymatic hydrolysis of pretreated coconut meal substrate with recombinant mannanase. Methods. Mannanase gene was cloned from Bacillus subtilis cAE24, then expressed in BL21. Purified Mannanase exhibit stability over a wide range of $\mathrm{pH}$ and temperature from $\mathrm{pH}$ 6-8 and 4 ${ }^{\circ}$ Celsius to $70^{\circ} \mathrm{Celsius}$, respectively. SEM analysis revealed that sonication could change the surface characteristic of copra meal, which gave better MOS yield, compared to untreated substrates. The separation and purification of each MOS were achieved using Biogel-P2 column chromatography. Determination of biological active MOS species was also investigated. T84 cells were cultured and treated with each of the purified MOS species to determine their tight junction enhancing activity.

Results. Scanning electron microscope imaging showed that pretreatment using sonication could disrupt the surface of copra meal better than grinding alone, which can improve the production of MOS. Pentamer of MOS (M5) significantly increased tight junction integration of

59 T84 cells measured with TEER $(p<0.0001)$.

Introduction supplement for increasing the life quality of pets and livestock. Several reports revealed that MOS supplement could improve growth performance and body weight in various animals(Ai et al. 2011; Dimitroglou et al. 2010; Genc et al. 2007; Mansour et al. 2012; Staykov et al. 2007). It can enhance the immunity and also metabolic and stress response of aquamarine cultures including shrimp, seabream, sturgeon and rainbow trout(Ai et al. 2011; Dimitroglou et al. 2010; Mansour et al. 2012; Ozaki et al. 2007; Rungrassamee et al. 2014; Staykov et al. 2007). Besides, the beneficial effects of MOS are observed in terrestrial animals like poultry and mammals as it raises nutrient digestibility, cecal fermentation, and improves the intestinal morphology(Cheled-Shoval et al. 2011; Cheled-Shoval et al. 2014; Dimitroglou et al. 2010; 
74 density and improve the organisation of the ileac villi This suggested a higher rate of intestinal

75 nutrient uptake; thus, improved the growth performance(Mourão et al. 2006). The researches

76 which were conducted in chickens, turkeys, pigs, and calves also demonstrated the similar

77 results(Che et al. 2012a; Che et al. 2011; Che et al. 2012b; Cheled-Shoval et al. 2011; Cheled-

78 Shoval et al. 2014; Corrigan et al. 2012; Ghosh \& Mehla 2012). Although the identification and

79 the mechanism of actions of MOS remained unclear, existing evidence strongly suggests that

MOS may exert biological effects on the intestine regarding tissue compact and increased in structure and activity.

MOS is often prepared by hydrolysis reaction of a mannose-contained glucan polymer, mainly glucomannan and galactomannan (Cescutti et al. 2002; Ganter et al. 1995; McCleary et al. 1976). Glucomannan, a soluble dietary fiber, is a heteropolymer chain of beta-D-glucose and beta-D-mannose which is partially attached with acetyl groups in a molar ratio of 1: 1.6 with beta 1-4 linkages. Glucomannan is widely distributed in the tuber or root of the konjac

(Amorphophallus konjac or Amorphophallus rivieri)(Maeda et al. 1980). Unfortunately, a recent study revealed that the oligosaccharides obtained from the digestion of glucomannan are composed of several random sequences of glucose and mannose residues which hinder the characterization of the oligosaccharides produced(Cescutti et al. 2002).

In contrast, galactomannan is an insoluble fiber found in the endosperm of many plant cell wall (Saccharomyces cerevisiae), commercially available as Bio-Mos ${ }^{\circledR}$ (Alltech, Inc., Nicholasville, KY). These types of mannan consist of (1-4)-beta-D-mannose repeating units with et al. 1976; Sittikijyothin et al. 2005). 
101 for an industrial scale guar gum production from cluster bean(Ganter et al. 1995; Miyazawa \& 102 Funazukuri 2006). Although this method was suitable for large-scale production, the sizes of 103 MOS produced cannot be predicted. MOS produced may also require additional purification 104 process to remove the acid and chemical contaminants. Hence, different types of substrate and reaction condition affect the characteristics of MOS product (Ganter et al. 1995)

Recently, the production of MOS by enzymatic hydrolysis is of great interested since the product obtained has become more specific and predictable. The enzyme mainly used in the process is beta-mannanase that was found in many organisms including fungus, yeast, and bacteria (Bourgault \& Bewley 2002; Chauhan et al. 2012; Cheng et al. 2016; Kim et al. 2018; Kurakake \& Komaki 2001; Larsson et al. 2006; Puchart et al. 2004; Rosengren et al. 2014; Shi et al. 2011; Talbot \& Sygusch 1990). Beta-mannosidase (E.C. 3.2.1.78) is a hemicellulose-type enzyme that catalyzes random hydrolysis of the internal beta-1,4-glycosidic bond of various mannan-type polymer yielding multiple size beta-1,4-mannooligosaccharides (Cescutti et al. 2002; Ghosh et al. 2015; Larsson et al. 2006; McCleary 1988; Talbot \& Sygusch 1990). Several studies have reported that enzymatic production could yield MOS with the degree of polymerisation from 2-6, which is related to those bioactive oligosaccharides that mostly have a low molecular weight or has a low to moderate degree of polymerisation(Mattaveewong et al. 2016; Muanprasat \& Chatsudthipong 2017; Muanprasat et al. 2015; Yousef et al. 2012). However, some enzymes produce a low amount of moderate size of MOS (DP 5 and above) from galactomannan substrate compared to those small sizes MOS (DP 2-4) (Ghosh et al. 2015; Rungrassamee et al. 2014).

Bacillus subtilis, a gram-positive bacterium, is well-known for its capability of secreting several beneficial enzymes such as beta-mannanase, xylanase, and glucanase. This bacterium can be isolated from soil, water, and decomposing plant matter. It has also been found in the gastrointestinal tract of animals (Hong et al. 2009; Lefevre et al. 2017; Tam et al. 2006; Wang \& Fung 1996). Bacillus subtilis is also used in a traditional fermented soybean food (e.g., Natto) in Japan (Lefevre et al. 2017; Wang \& Fung 1996). 
In this study, we reported the production of MOS from pretreated copra meal by

129

130

enzymatic hydrolysis using recombinant endo-1,4-beta mannosidase derived from Bacillus subtilis CAE24. Optimization of the enzymatic production and purification of MOS was elucidated. The screening of bioactive MOS was performed by measuring the effects of of each purified MOS oligomers treatment on a tight junction assembly of an intestinal epithelial cell line.

\section{Materials \& Methods}

\section{Substrate preparation}

Galactomannan substrate was received as a dry granulated copra meal, a residual waste from coconut milk and oil extraction process. Forty grams of copra meal was ground using a high-power blender (Moulinex, France). A finely ground copra meal powder was then subjected to solvent extraction to remove the remaining oil with 200 milliliters of $n$-hexane, and with sonication for 10 minutes. The suspension was filtered with a cellulose filter paper (Whatman no.1, Sigma Aldrich, USA.). This process was repeated 3 times before the copra meal was left to dry in an oven at $60{ }^{\circ} \mathrm{C}$ overnight. The dried substrate was resuspended in 400 milliliters of deionized water and then autoclaved at $110{ }^{\circ} \mathrm{C}$ and $15 \mathrm{psi}$ for 20 minutes. The product of this step is labelled as G-GalMan.

Thereafter, G-GalMan was sonicated using a probe-type sonicator at $40 \%$ power, 2seconds/1second pulse, for 300 minutes. The sonicated products were spun down at 10,000xg and washed with deionized water twice before resuspending in 100 milliliters of $0.05 \mathrm{M}$ citrate buffer $\mathrm{pH}$ 6.5. The final pretreated product is labelled as S-GalMan. Wet weight and dry weight of S-GalMan were measured.

\section{Structural analysis of pretreatment galactomannan substrate}

All the deoiled substrates, including G-GalMan, S-Galman, and digested products were prepared as previously described. All the substrates were subjected to critical point dryer (CPD) 
154 and were coated with gold particles prior to the scanning electron microscopy (SEM-EDS) (Jeol 155 JSM-6400 scanning electron microscope, Jeol Ltd. Japan).

Isolation of Bacillus subtilis cAE24

A baiting technique, burying dry copra meal in the soil at different suitable locations, was

performed for the isolation of mannanase-produced bacteria. The bacteria were dispersed from the buried copra meal with $3 \mathrm{~mL}$ of sterile distilled water, then serially diluted and screened on minimal medium plates containing $1 \%$ glucomannan as a carbon source. The positive colony, with clear zone, were then picked and used for enzyme production in minimal medium containing $1 \%$ glucomannan, $0.03 \%$ magnesium sulfate, $0.1 \%$ ammonium sulfate, $0.6 \%$ potassium dihydrogenphosphate, and $1 \%$ potassium hydrogenphosphate. The crude enzyme produced in the supernatant was collected. Mannanase activity was assayed using Dinitrosalicylic (DNS) colorimetric assay with $0.8 \%$ glucomannan as a substrate (Miller 1959; Vanaja \& Shobha Rani 2007). The colony with the highest mannanase activity was collected. It was identified by 16 s rRNA sequence comparison method.

\section{Cloning and construction of recombinant mannanase}

The amplification of target mannanase gene was accomplished by using information from the genome database il433616933:611507-613595 of Bacillus subtilis strain BEST7613. The polymerase chain reaction was performed using 5'-GGGGAGTTGCATATGTTTAAGA-3' and 5'GCGGAACGTCTGATTAGAGC-3' as a forward primer and reverse primer, respectively. The product encoding mannanase gene was sequenced and submitted to NCBI. DNA sequencing data is available at NCBI via GenBank accession number KY951415. The PCR product was with $\mathrm{Xhol}$ and $\mathrm{Ndel}$ restriction endonuclease, and the mannanase gene was subcloned into 
181

182

183

184

185

186

187

188

189

190

191

192

193

194

195

196

197

198

199

200

201

202

203

204

205

206

207

BL21 (DE3) expression host (Novagen, USA). This pRM24 containing E. coli BL21 (DE3) was named RM24.

\section{Purification and Biochemical characterization of RMase24}

The crude enzyme production was done with RM24 cultured in $1 x$ LB at $37{ }^{\circ} \mathrm{C}$ with 1.0 mM IPTG. Mannanase, RMase24, was collected as extracellular enzyme and concentrated before dialyzing against $20 \mathrm{mM}$ tris- $\mathrm{HCl}$ buffer $\mathrm{pH} 7.5$ at $4{ }^{\circ} \mathrm{C}$ and then purified through $\mathrm{DEAE}$ toyopearl column. The fractions with enzymatic activity were pooled together. Ammonium sulfate was added to give the final concentration of 1 then it was applied onto phenyl toyopearl column. A linear salt gradient from 1.0 - $0 \mathrm{M}$ ammonium sulfate in $20 \mathrm{mM}$ Tris- $\mathrm{HCl} \mathrm{pH} 7.5$ was applied. Fractions that possessed RMase24 activity were collected and pooled. The purity of the enzyme was determined by SDS-PAGE. The activity of RMase 24 was assayed by measuring the total reducing sugar using DNS colourimetric method (Miller 1959). The activity unit was defined as the amount of enzyme that is required to release 1 micromole of reducing sugar from $0.5 \% \mathrm{w} / \mathrm{v}$ glucomannan substrate per minute.

The optimum $\mathrm{pH}$ and temperature of purified RMase24 were also explored using $0.4 \% \mathrm{w} /$ v glucomannan. The optimal $\mathrm{pH}$ for RMase24 was measured in a $\mathrm{pH}$ range of $2.5-10.0$ at $70{ }^{\circ} \mathrm{C}$ in $50 \mathrm{mM}$ glycine-HCl buffer $(\mathrm{pH}$ 2.5-3.5), citrate buffer (pH 3.5-6.0), phosphate buffer ( $\mathrm{pH}$ 6.08.0) or glycine- $\mathrm{NaOH}$ (8.0-10.0). The optimum temperature for RMase24 was determined by assaying enzymatic activity in $50 \mathrm{mM}$ citrate buffer $\mathrm{pH} 6.0$ in a temperature range of $4-80^{\circ} \mathrm{C}$. For pH stability analysis, the residual enzymatic activity was measured by preincubating the enzyme at $4{ }^{\circ} \mathrm{C}$ for $24 \mathrm{~h}$ at various $\mathrm{pH}$ described above. The thermostability of RMase 24 was determined by measuring the residual activity of the enzyme after incubation in $50 \mathrm{mM}$ citrate buffer $\mathrm{pH} 6.0$ at $70{ }^{\circ} \mathrm{C}$ from 0 to $6 \mathrm{~h}$. The experiment was performed triplicate. Each data point is the mean, while the error bar is the standard deviation.

Peer] reviewing PDF | (2019:01:34726:1:2:NEW 22 May 2019) 
208

209

210

211

212

213

\section{Effects of metal ions and some chemical reagent on the activity of RMase24}

The effect of metal ions and some chemical reagent on the activity of RMase24 was determined by measuring the mannanase activity in a reaction containing $0.5 \%$ glucomannan, $50 \mathrm{mM}$ citrate buffer (pH 6.0) and $5 \mathrm{mM}$ of some ions including $\mathrm{Na}^{+}, \mathrm{K}^{+}, \mathrm{Mg}^{2+}, \mathrm{Co}^{2+}, \mathrm{Ca}^{2+}, \mathrm{Mn}^{2+}$, $\mathrm{Cu}^{2+}, \mathrm{Zn}^{2+}, \mathrm{Fe}^{3+}$, and EDTA) or $0.1 \%(\mathrm{w} / \mathrm{v})$ of surfactants including SDS and TritonX-100 at $70^{\circ} \mathrm{C}$. The activity was measured using DNS assay using glucose as a standard(Miller 1959).

\section{Production of MOS from a crude RMase24}

The digestion of S-GalMan and G-GalMan with crude RMase24 was performed. One hundred micrograms of each substrate were used in a process with various enzyme/substrate concentration ranging from 1 unit to 100 units per 1 gram of substrate. After the reactions were incubated for 24 hours at $37^{\circ} \mathrm{C}$, digestion mixtures were centrifuged at $10,000 \mathrm{~g}$ to remove the undigested debris. The hydrolysate was filtered using filter paper (Whatman cellulose filter paper grade 4, Sigma Aldrich, USA) and 0.45 micrometers cellulose acetate membrane filter (Whatman cellulose acetate membrane, Sigma Aldrich, USA). Crude MOS produced from each substrate was collected then lyophilized and measured the dry-weight. The percentage yield was calculated to compare the efficiency between each type of substrate pretreatment.

The soluble MOS product was purified using Biogel-P2 size exclusion column chromatography. The collected MOS fractions were then lyophilized and analyzed using thin layer chromatography (TLC) using acetic: butanol: water solvent system of 3: 3: 2 as mobile phase at $35-40{ }^{\circ} \mathrm{C}$. The separated MOS was visualized by orcinal-sulfuric acid staining solution. The molecular mass determination of tetramer to heptamer MOS was performed using MALDITOF mass spectrometer (Solari X, FT mass spectrometry, Bruker, United States).

\section{Determination and identification of bioactive MOS}



micrometer Whatman uniflo syringe filter (GE healthcare, USA) before being made up to 10 micromolar solutions with Dulbecco's Modified Eagle's Medium (DMEM, Corning life science, resistance method (TEER) method, with and without $\mathrm{Ca}^{2+}$ switch assay. The measurement protocol was described in detail in our previous study (Muanprasat et al. 2015). The cell line used in this experiment was T84, a lung metastasized-human colonic adenocarcinoma cell. The cell was growth in DMEM with $10 \%$ fetal bovine serum in 12mm. Transwell (Corning, USA) culture plates and measured the TEER daily. Once the electrical resistant of the cells became steady, each prepared MOS was treated to the cells. The change in TEER was monitored at 24 hours post-treatment. The tight junction reassembling assay was performed with $\mathrm{Ca}^{2+}$ switch assay. T84 cells were cultured in DMEM medium until the population of the cells reached 85\% confluency on transwell plate, then DMEM medium was substituted with SMEM to remove $\mathrm{Ca}^{2+}$ ions. After 24-hour incubation, SMEM was then substituted with prepared DMEM-M5. TEER at different time intervals were measured to monitor the reassembling of the tight junction for 12

249 hours.

\section{Statistical analysis}

Statistical analysis was performed using GraphPad prism7 (GraphPad Software Inc. CA,

\section{Results}

\section{Isolation of Bacillus subtilis cAE24 and cloning of RMase24 gene}

We have successfully isolated bacterial strain with high mannanase activity. It was 
261 available in Supplementary Files (Data S1 and Figure S1). The gene sequence of RMase24 262 was 1090 bp in size, encoding 362 amino acid residues (Data S2). The theoretical molecular 263 weight and pl is 40,918.08 Da and 5.80, respectively. The amino acid sequence of RMase24

264 was blasted with NCBI database and aligned with mannanase sequences from various Bacillus 265 subtilis strain. The alignment result showed 99\% identity. Three-dimensional structure of 266 RMase24 was constructed using Swiss-PdbViewer, DeepView version 4.1, compared with 267 mannan endo-1,4-beta- mannosidase from Bacillus subtilis BEST7613 (accession number 268 BAM49507.1). The sequence alignment and the 3D structure are available in Supplementary 269 Files (Figure S2 and S3).

\section{Production and characterization of RMase24}

A recombinant mannanase gene from Bacillus subtilis cAE24 was successfully cloned and expressed. The recombinant enzyme was named RMase24. The crude enzyme was purified through DEAE Toyopearl DEAE650m and Toyopearl phenyl 650m column chromatography, respectively. The specific activity of RMase24 increased a 3.1 fold, 1800 unit per milligram protein. RMase24 possesses the ability to work under a wide $\mathrm{pH}$ range. RMase24 had over $80 \%$ of its maximum activity at $\mathrm{pH}$ ranging from 5.0 to 8.0 with the optimum activity in citrate buffer $\mathrm{pH} 6.0$ (figure 1A.). RMase24 has over $80 \%$ activity from 45 to $70{ }^{\circ} \mathrm{C}$, with the optimum temperature at $70{ }^{\circ} \mathrm{C}$. (Figure 1B.) Thus, the broad spectrum of $\mathrm{pH}$ and temperature of RMase24 made it possible to set up the reaction under various desirable reaction conditions. Interestingly, purified RMase24 was stable, retaining most of its activity, when stored under alkali condition. The stability of RMase24 significantly dropped when the $\mathrm{pH}$ of the storage buffer became more acidic (figure 1C). minutes. (figure 1D) 

enhancing effects to RMase 24 were cobalt and zinc ions, which increased its activity to about agents also affected the activity of RMase24. The reaction contained SDS or EDTA decreases the activity of RMase 24 to $25 \%$ and $44 \%$, respectively. While Triton X-100 had no effects on RMase24 activity. (figure1E)

\section{Structural analysis of pretreatment galactomannan substrates}

The surface structure of each substrate was analyzed by scanning electron microscopy.

The result showed different characteristics of fragment surface, edges and size distribution.

Sonication pretreatment gave smaller average fragment size when compared with the untreated substrate (figure 2A-B). At higher magnification, different characters of fragment surface and edges from each type of substrate was observed. (figure 2C-D). S-GalMan had more irregular amorphous shape edges, and there was a considerable amount of amorphous structure on the surface of the substrate, while G-GalMan has less of these characteristics. Interestingly, after the digestion of S-GalMan with RMase24, these small fragments were reduced, and the edge and surface of the fragments became smooth. In contrast, digestion of G-GalMan did not change the overall structure observed by SEM much (figure 2E-F)

\section{MOS production and purification, the effect of substrate pretreatment on the production,} and the structural prediction of M5

The percentages yield of total MOS produced from S-GalMan and G-GalMan were products showed a spectrum of MOS with size ranging from DP2 to DP6 and MOS with a size 
314 when compared to G-GalMan at every incubation period. S-GalMan could be digested more 315 readily than G-GalMan, liberating a higher amount of MOS, within the first 6 hours. (figure 3A). 316 Determination of appropriate RMase24 units required for optimum MOS production was 317 then performed with S-GalMan to determine the optimal enzyme/substrate ratio. The results 318 showed that the enzyme/substrate ratio used in the digestion affected the pattern of MOSs 319 produced (figure $3 C$ ). The enzyme-substrate ratio of 10 unit per 1 gram of dried substrate showed the highest yield of MOS DP4 to DP6. Interestingly, at higher concentration of RMase24 we observed a reduction of DP6 and DP9. (figure 3B).

M5 affect the tight junction integration of an epithelial tissue

MOS DP4 (M4) to DP7 (M7) was successfully purified through Biogel-P2 column (figure

4A) and their molecular mass confirmed by mass spectrometry. The arrow in figure 4C-F indicated a peak of molecular mass of each MOS with sodium ion; M4- $\mathrm{Na}^{+}=679.123 \mathrm{~m} / \mathrm{z}$, M5$\mathrm{Na}^{+}=851.2646 \mathrm{~m} / \mathrm{z}, \mathrm{M} 6-\mathrm{Na}^{+}=1013.3171 \mathrm{~m} / \mathrm{z}, \mathrm{M} 7-\mathrm{Na}^{+}=1175.3700 \mathrm{~m} / \mathrm{z}$, respectively. Tight junction integration was measured on T84 cells treated with M4 to M7 using TEER assay. The result demonstrated that treating the cells with M5 significantly increase the relative percentage of TEER $(p<0.0001)$. This demonstrated that M5 can enhance tight junction integration of T84 cell while treating cells with M4, M6 and M7 did not demonstrate any significant effect on TEER comparing with vehicle (figure5A). The effects of M5 over cellular tight junction was distinguished from the cellular proliferation by calcium depletion experiment where calcium ions were removed from the culture medium once the growth of the cell reached $85 \%$ confluency, replacing DMEM with SMEM. After 24 hours of incubation in SMEM, the culture medium was without M5 (figure 5B). 
341

342

343

344

345

346

347

348

349

350

351

352

353

354

355

356

357

358

359

360

361

362

363

364

365

\section{Discussion}

In this study, recombinant mannanase, RMase24 together with a proper substrate pretreatment and enzyme-substrate ratio showed a better production yield of moderate MOSs compared with previous studies (Ghosh et al. 2015; Rungrassamee et al. 2014). These results may result from the higher exposed surface area arose from the additional sonication step that is accessible to enzymatic hydrolysis. Interestingly, the enzyme-substrate ratio affected the yield and size distribution of MOS products. We observed a reduction in the band intensity of M4, M6, and M9, while Intensity of other bands including M2, M3, and M5 increased as the enzyme over substrate ratio increases, figure3C. At high enzyme over substrate ratio, the availability free enzyme in the solution may cause further hydrolysis of some MOS products that remains a good substrate for the enzyme. These results indicate that the substrate specificity of RMase24 is different for each MOS species being produced.

Biological activity of MOS have been previously reported (Che et al. 2012a; Che et al. 2011; Che et al. 2012b; Cheled-Shoval et al. 2011; Cheled-Shoval et al. 2014; Corrigan et al. 2012; Dimitroglou et al. 2010; Genc et al. 2007; Ghosh et al. 2015; Ghosh \& Mehla 2012; Grisdale-Helland et al. 2008; Mansour et al. 2012; Mourão et al. 2006; Staykov et al. 2007). However, these studies used a mixture of MOS of different sizes. Thus, the biologically active MOS species were not identified. In this study, we separated and purified each MOS species prior to biological activity analysis. We discovered that M5 was the only molecule that significantly enhanced the tight junction integration of T84 cells. This effect was distinguished from the effect of cell proliferation by $\mathrm{Ca}^{2+}$ switch assay (Figure5B). This finding indicated that M5 was playing the vital role in increasing the tight junction of epithelial tissue. Hereafter, further investigation is required to identify the structure and mechanism of action of M5.

Conclusions 
using proper substrate pre-treatment. The enzyme-substrate ratio used for MOS production is a

\section{References}

Ai Q, Xu H, Mai K, Xu W, Wang J, and Zhang W. 2011. Effects of dietary supplementation of Bacillus subtilis and fructooligosaccharide on growth performance, survival, non-specific immune response and disease resistance of juvenile large yellow croaker, Larimichthys crocea. Aquaculture 317:155-161.

Bourgault R, and Bewley JD. 2002. Variation in its C-terminal amino acids determines whether endo- $\beta$-mannanase is active or inactive in ripening tomato fruits of different cultivars. Plant physiology 130:1254-1262.

Cescutti P, Campa C, Delben F, and Rizzo R. 2002. Structure of the oligomers obtained by enzymatic hydrolysis of the glucomannan produced by the plant Amorphophallus konjac. Carbohydrate Research 337:2505-2511.

Chauhan PS, Puri N, Sharma P, and Gupta N. 2012. Mannanases: microbial sources, production, properties and potential biotechnological applications. Applied microbiology and biotechnology 93:1817-1830.

Che T, Johnson R, Kelley K, Dawson K, Moran C, and Pettigrew J. 2012a. Effects of mannan oligosaccharide on cytokine secretions by porcine alveolar macrophages and serum cytokine concentrations in nursery pigs. Journal of animal science 90:657-668.

Che T, Johnson R, Kelley K, Van Alstine W, Dawson K, Moran C, and Pettigrew J. 2011. Mannan oligosaccharide modulates gene expression profile in pigs experimentally infected with porcine reproductive and respiratory syndrome virus. Journal of animal science 89:3016-3029.

Che T, Song M, Liu Y, Johnson R, Kelley K, Van Alstine W, Dawson K, and Pettigrew J. 2012b. Mannan oligosaccharide increases serum concentrations of antibodies and inflammatory mediators in weanling pigs experimentally infected with porcine reproductive and respiratory syndrome virus. Journal of Animal Science 90:2784-2793.

Cheled-Shoval S, Amit-Romach E, Barbakov M, and Uni Z. 2011. The effect of in ovo administration of mannan oligosaccharide on small intestine development during the preand posthatch periods in chickens. Poultry science 90:2301-2310.

Cheled-Shoval S, Gamage NW, Amit-Romach E, Forder R, Marshal J, Van Kessel A, and Uni Z. 2014. Differences in intestinal mucin dynamics between germ-free and conventionally reared chickens after mannan-oligosaccharide supplementation. Poultry science 93:636644.

Cheng L, Duan S, Feng X, Zheng K, Yang Q, and Liu Z. 2016. Purification and characterization of a thermostable $\beta$-mannanase from Bacillus subtilis BE-91: potential application in inflammatory diseases. BioMed research international 2016. 
408

409

410

411

412

413

414

415

416

417

418

419

420

421

422

423

424

425

426

427

428

429

430

431

432

433

434

435

436

437

438

439

440

441

442

443

444

445

446

447

448

449

450

451

452

453

454

455

456

457

458

459
Corrigan A, Horgan K, Clipson N, and Murphy R. 2012. Effect of dietary prebiotic (mannan oligosaccharide) supplementation on the caecal bacterial community structure of turkeys. Microbial ecology 64:826-836.

Dimitroglou A, Merrifield DL, Spring P, Sweetman J, Moate R, and Davies SJ. 2010. Effects of mannan oligosaccharide (MOS) supplementation on growth performance, feed utilisation, intestinal histology and gut microbiota of gilthead sea bream (Sparus aurata). Aquaculture 300:182-188.

Ganter JLM, Heyraud A, Petkowicz CL, Rinaudo M, and Reicher F. 1995. Galactomannans from Brazilian seeds: characterization of the oligosaccharides produced by mild acid hydrolysis. International journal of biological macromolecules 17:13-19.

Genc M, Aktas M, Genc E, and Yilmaz E. 2007. Effects of dietary mannan oligosaccharide on growth, body composition and hepatopancreas histology of Penaeus semisulcatus (de Haan 1844). Aquaculture Nutrition 13:156-161.

Ghosh A, Verma AK, Tingirikari JR, Shukla R, and Goyal A. 2015. Recovery and purification of oligosaccharides from copra meal by recombinant endo- $\beta$-mannanase and deciphering molecular mechanism involved and its role as potent therapeutic agent. Molecular biotechnology 57:111-127.

Ghosh S, and Mehla RK. 2012. Influence of dietary supplementation of prebiotics (mannanoligosaccharide) on the performance of crossbred calves. Tropical animal health and production 44:617-622.

Grisdale-Helland B, Helland SJ, and Gatlin III DM. 2008. The effects of dietary supplementation with mannanoligosaccharide, fructooligosaccharide or galactooligosaccharide on the growth and feed utilization of Atlantic salmon (Salmo salar). Aquaculture 283:163-167.

Hong HA, Khaneja R, Tam NM, Cazzato A, Tan S, Urdaci M, Brisson A, Gasbarrini A, Barnes I, and Cutting SM. 2009. Bacillus subtilis isolated from the human gastrointestinal tract. Research in microbiology 160:134-143.

Kim S, Lee M-H, Lee E-S, Nam Y-D, and Seo D-H. 2018. Characterization of mannanase from Bacillus sp., a novel Codium fragile cell wall-degrading bacterium. Food Science and Biotechnology 27:115-122. 10.1007/s10068-017-0210-3

Kurakake $M$, and Komaki T. 2001. Production of $\beta$-mannanase and $\beta$-mannosidase from Aspergillus awamori K4 and their properties. Current microbiology 42:377-380.

Larsson AM, Anderson L, Xu B, Muñoz IG, Usón I, Janson J-C, Stålbrand H, and Ståhlberg J. 2006. Three-dimensional crystal structure and enzymic characterization of $\beta$-mannanase Man5A from blue mussel Mytilus edulis. Journal of molecular biology 357:1500-1510.

Lefevre M, Racedo SM, Denayrolles M, Ripert G, Desfougères T, Lobach AR, Simon R, Pélerin $F$, Jüsten $P$, and Urdaci MC. 2017. Safety assessment of Bacillus subtilis CU1 for use as a probiotic in humans. Regulatory Toxicology and Pharmacology 83:54-65.

Maeda M, Shimahara H, and Sugiyama N. 1980. Detailed examination of the branched structure of konjac glucomannan. Agricultural and Biological Chemistry 44:245-252.

Mansour MR, Akrami R, Ghobadi S, Denji KA, Ezatrahimi N, and Gharaei A. 2012. Effect of dietary mannan oligosaccharide (MOS) on growth performance, survival, body composition, and some hematological parameters in giant sturgeon juvenile (Huso huso Linnaeus, 1754). Fish physiology and biochemistry 38:829-835.

Mathur N. 2016. Industrial galactomannan polysaccharides: CRC Press.

Mattaveewong T, Wongkrasant $\mathrm{P}$, Chanchai S, Pichyangkura R, Chatsudthipong $\mathrm{V}$, and Muanprasat C. 2016. Chitosan oligosaccharide suppresses tumor progression in a mouse model of colitis-associated colorectal cancer through AMPK activation and suppression of NF-KB and mTOR signaling. Carbohydrate polymers 145:30-36.

McCleary B, Matheson N, and Small DM. 1976. Galactomannans and a galactoglucomannan in legume seed endosperms: Structural requirements for $\beta$-mannanase hydrolysis.

Phytochemistry 15:1111-1117.

McCleary BV. 1988. ß-D-Mannanase. Methods in Enzymology: Elsevier, 596-610. 
460

461

462

463

464

465

466

467

468

469

470

471

472

473

474

475

476

477

478

479

480

481

482

483

484

485

486

487

488

489

490

491

492

493

494

495

496

497

498

499

500

501

502

503

504

505

506

507

508

509

510
Miller GL. 1959. Use of dinitrosalicylic acid reagent for determination of reducing sugar. Analytical chemistry 31:426-428.

Miyazawa T, and Funazukuri T. 2006. Noncatalytic hydrolysis of guar gum under hydrothermal conditions. Carbohydrate research 341:870-877.

Mourão JL, Pinheiro V, Alves A, Guedes C, Pinto L, Saavedra MJ, Spring P, and Kocher A. 2006. Effect of mannan oligosaccharides on the performance, intestinal morphology and cecal fermentation of fattening rabbits. Animal Feed Science and Technology 126:107120.

Muanprasat C, and Chatsudthipong V. 2017. Chitosan oligosaccharide: Biological activities and potential therapeutic applications. Pharmacology \& therapeutics 170:80-97.

Muanprasat C, Wongkrasant P, Satitsri S, Moonwiriyakit A, Pongkorpsakol P, Mattaveewong T, Pichyangkura R, and Chatsudthipong V. 2015. Activation of AMPK by chitosan oligosaccharide in intestinal epithelial cells: Mechanism of action and potential applications in intestinal disorders. Biochemical pharmacology 96:225-236.

Ozaki K, Fujii S, and Hayashi M. 2007. Effect of dietary mannooligosaccharides on the immune system of ovalbumin-sensitized mice. Journal of health science 53:766-770.

Puchart V, Vršanská M, Svoboda P, Pohl J, Ögel ZB, and Biely P. 2004. Purification and characterization of two forms of endo- $\beta-1$, 4-mannanase from a thermotolerant fungus, Aspergillus fumigatus IMI 385708 (formerly Thermomyces lanuginosus IMI 158749). Biochimica et Biophysica Acta (BBA)-General Subjects 1674:239-250.

Rosengren A, Reddy SK, Sjöberg JS, Aurelius O, Logan DT, Kolenová K, and Stålbrand H. 2014. An Aspergillus nidulans $\beta$-mannanase with high transglycosylation capacity revealed through comparative studies within glycosidase family 5. Applied microbiology and biotechnology 98:10091-10104.

Rungrassamee W, Kingcha Y, Srimarut Y, Maibunkaew S, Karoonuthaisiri N, and Visessanguan W. 2014. Mannooligosaccharides from copra meal improves survival of the Pacific white shrimp (Litopenaeus vannamei) after exposure to Vibrio harveyi. Aquaculture 434:403410.

Shi P, Yuan T, Zhao J, Huang H, Luo H, Meng K, Wang Y, and Yao B. 2011. Genetic and biochemical characterization of a protease-resistant mesophilic $\beta$-mannanase from Streptomyces sp. S27. Journal of industrial microbiology \& biotechnology 38:451-458.

Sittikijyothin W, Torres D, and Gonçalves M. 2005. Modelling the rheological behaviour of galactomannan aqueous solutions. Carbohydrate Polymers 59:339-350.

Staykov Y, Spring P, Denev S, and Sweetman J. 2007. Effect of a mannan oligosaccharide on the growth performance and immune status of rainbow trout (Oncorhynchus mykiss). Aquaculture International 15:153-161.

Talbot G, and Sygusch J. 1990. Purification and characterization of thermostable betamannanase and alpha-galactosidase from Bacillus stearothermophilus. Appl Environ Microbiol 56:3505-3510.

Tam NK, Uyen NQ, Hong HA, Duc LH, Hoa TT, Serra CR, Henriques AO, and Cutting SM. 2006. The intestinal life cycle of Bacillus subtilis and close relatives. Journal of bacteriology 188:2692-2700.

Vanaja K, and Shobha Rani R. 2007. Design of experiments: concept and applications of Plackett Burman design. Clinical research and regulatory affairs 24:1-23.

Wang J, and Fung DY. 1996. Alkaline-fermented foods: a review with emphasis on pidan fermentation. Critical Reviews in Microbiology 22:101-138.

Yousef M, Pichyangkura R, Soodvilai S, Chatsudthipong V, and Muanprasat C. 2012. Chitosan oligosaccharide as potential therapy of inflammatory bowel disease: therapeutic efficacy and possible mechanisms of action. Pharmacological research 66:66-79. 
Figure 1

Optimization, characteristic of purified RMase24.

(A) and (B) reveal the optimum $\mathrm{pH}$ and temperature of purified RMase24, respectively. (C) represent the $\mathrm{pH}$ stability of RMase 24 under a storage at $4 \mathrm{C}$ for 24 hours. (D) represent thermal stability of RMase24 under a certain temperature for a period of time.
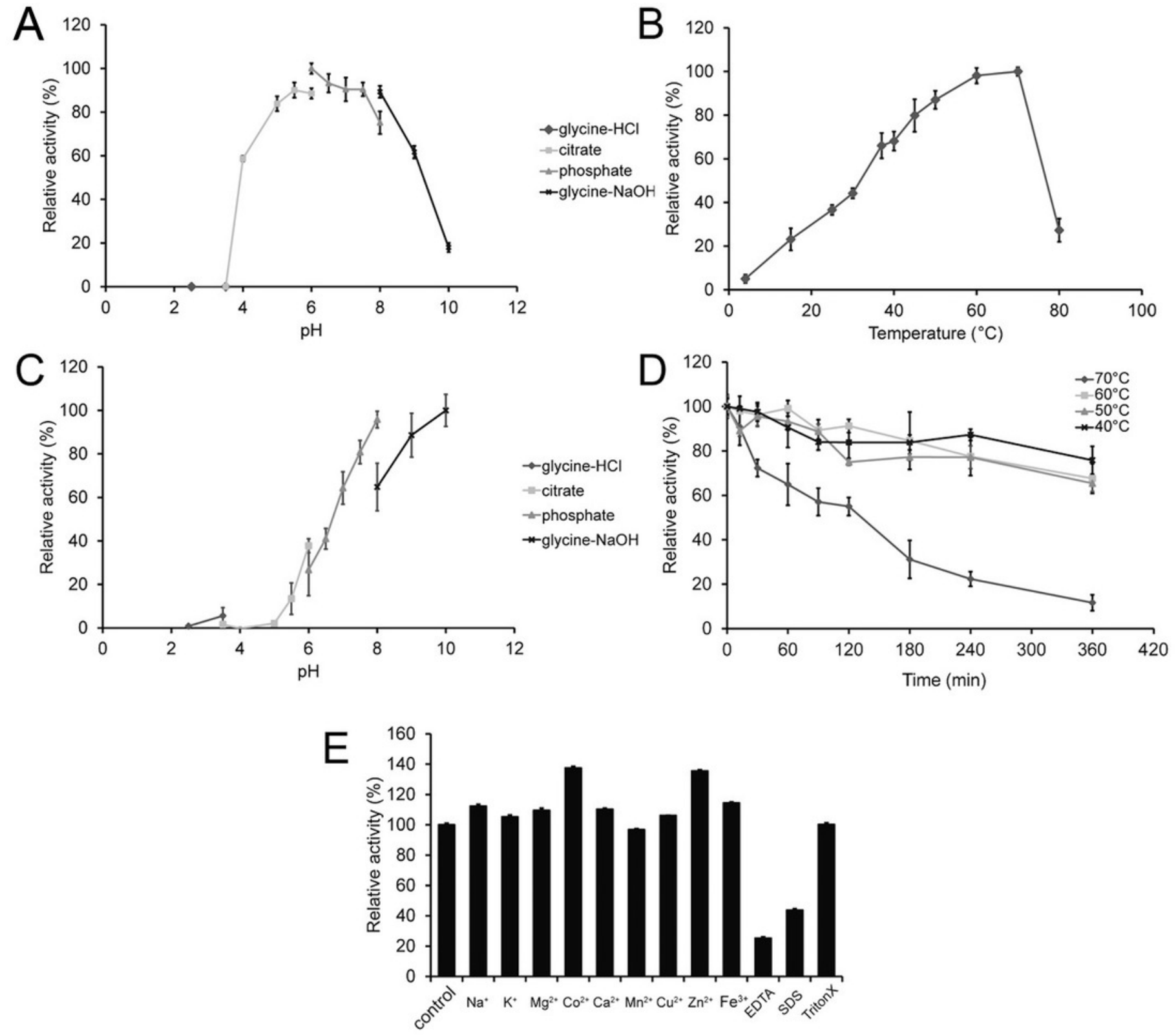


\section{Figure 2}

SEM analysis of S-GalMan and G-GalMan.

(A) and (B) represent the average fragment size of coconut meal of G-GalMan and S-GalMan, respectively at $\times 100$ magnification. Panel (C) and (D) show the characteristic of the fragment edge and surface of G-GalMan and S-GalMan, respectively. Panel (E) and (F) show the edge and surface structureof G-GalMan and S-GalMan, respectively, after a digestion.

*Note: Auto Gamma Correction was used for the image. This only affects the reviewing manuscript. See original source image if needed for review.

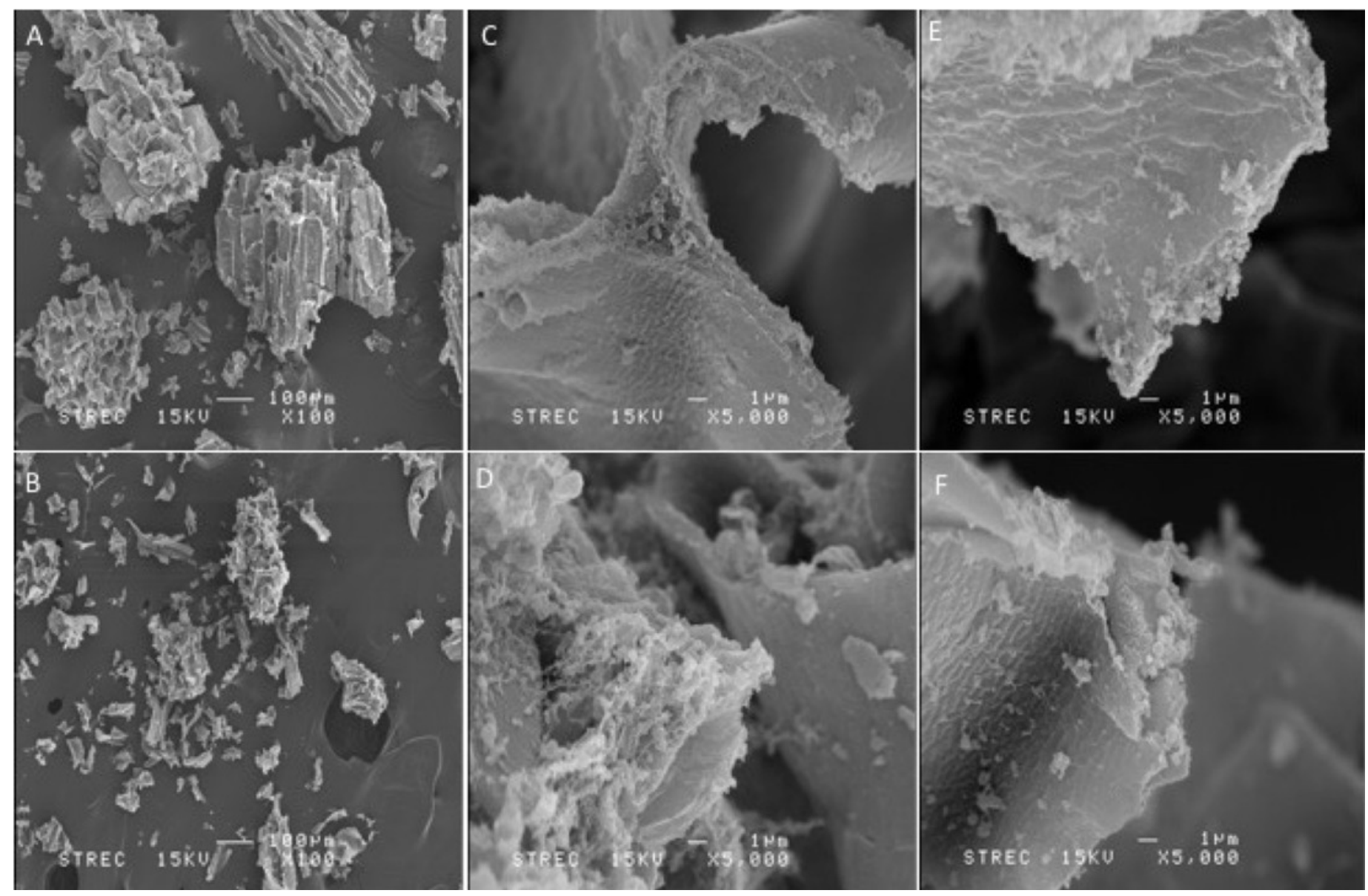




\section{Figure 3}

Product spectrum at different incubation time and enzyme substrate ratio.

A comparison between a digestion of S-GalMan (A) and G-GalMan (B) with 10 unit per $1 \mathrm{gram}$ of substrate. (ML; Mannan oligosaccharides ladder, No; no RMase24 in the reaction, m1-m6 represents mannose to mannohexose, respectively) (C) The pattern of MOS production at different RMase24 and substrate ratio, the substrate was fixed at $1 \mathrm{gram}$. (GL; Glucooligosaccharides ladder, g1-g7 represent glucose to glucoheptose, respectively) 
A $\mathrm{m} 1$

m2

m3

m4

$\mathrm{m} 5$

$\mathrm{m} 6$

\section{ML no $0 \mathrm{hr} 6 \mathrm{hr} 12 \mathrm{hr} 18 \mathrm{hr} 24 \mathrm{hr} 48 \mathrm{hr} \mathrm{ML}$ enz.}

B $\mathrm{m} 1$

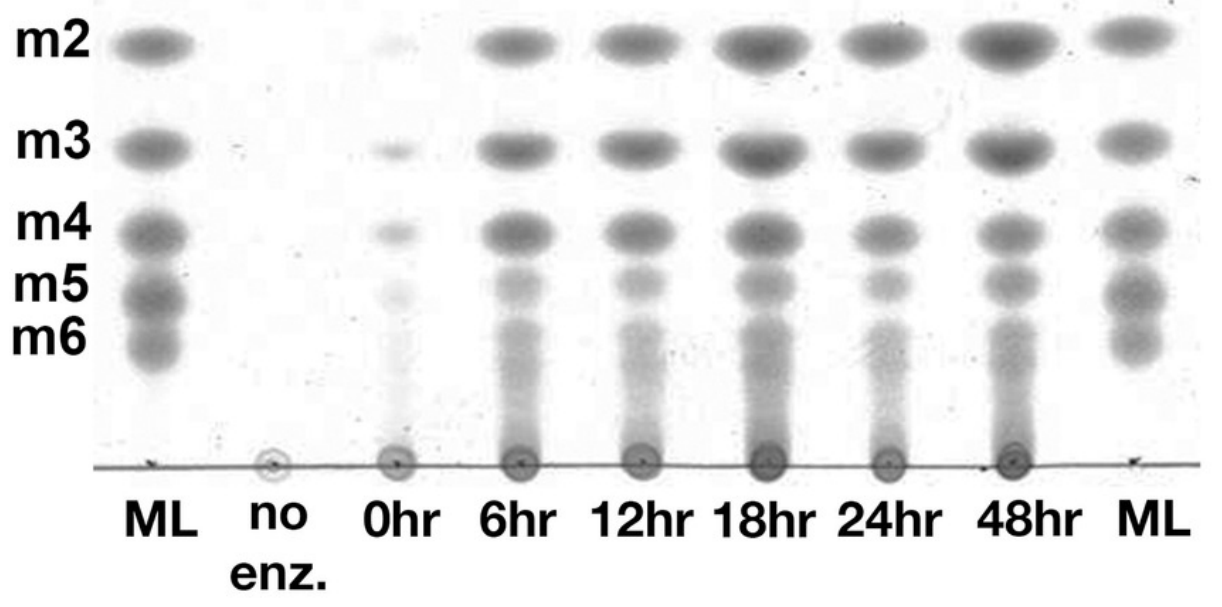

C

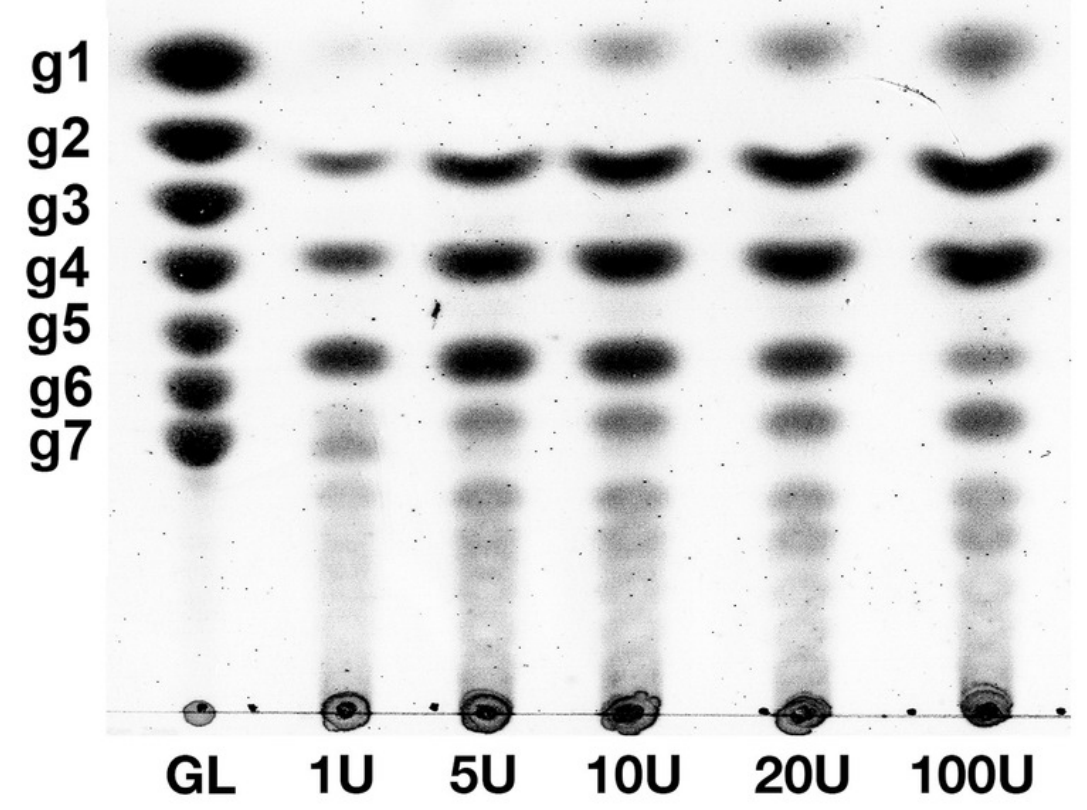




\section{Figure 4}

Purified MOS, from M4 to M7.

(A) Purified MOS, from M4 to M7 analyzed on TLC. ML stands for Mannooligosaccharides ladder, m1-m5 represent standard mannose to mannopentose, respectively. (B) Highperformance anion exchange chromatography (HPAEC-PAD) chromatogram of purified M5. The black arrow indicates the peak of M5. (C-F) High-resolution mass spectrometry of purified M4, M5, M6, and M7, respectively. The arrow indicated a peak of molecular mass of each MOS with sodium ion; $\mathrm{M} 4-\mathrm{Na}=679.123 \mathrm{~m} / \mathrm{z}, \mathrm{M} 5-\mathrm{Na}=851.2646 \mathrm{~m} / \mathrm{z}, \mathrm{M} 6-\mathrm{Na}=1013.3171$ $\mathrm{m} / \mathrm{z}, \mathrm{M} 7-\mathrm{Na}=1175.3700 \mathrm{~m} / \mathrm{z}$. 

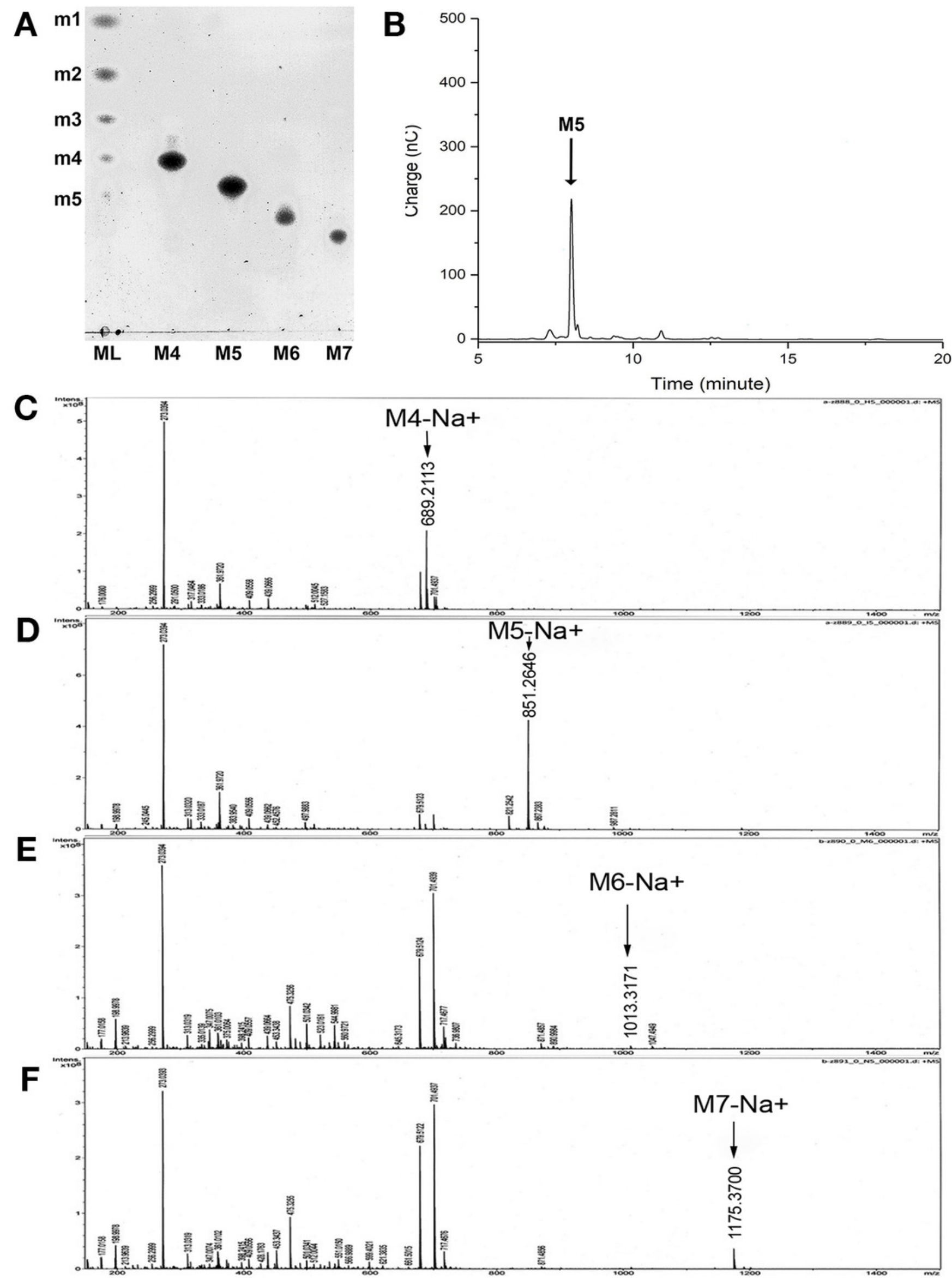

Peer) reviewing PDF | (2019:01:34726:1:2:NEW 22 May 2019) 
Figure 5

Effects of MOS on tight junsssction integration of T84 cells.

(A) Transepithelial electrical resistant (TEER) result of T84 cells treated with 10 micromolar of purified M4 to M7, compared to vehicle (non-treatment group). M5 significantly increased TEER of T84 cells (one-way ANOVA, $p<0.00001, n=3$ ). (B) Determination of tight junction reassembly of T84 affected by a treatment of 10 micromolar M5 compared to vehicle using $\mathrm{Ca}^{2+}$ swtich assay. M5 significantly recovered TEER of T84 cells after the destruction of the tight junction. (two-way ANOVA, $p<0.00001, n=4 s$ ). 


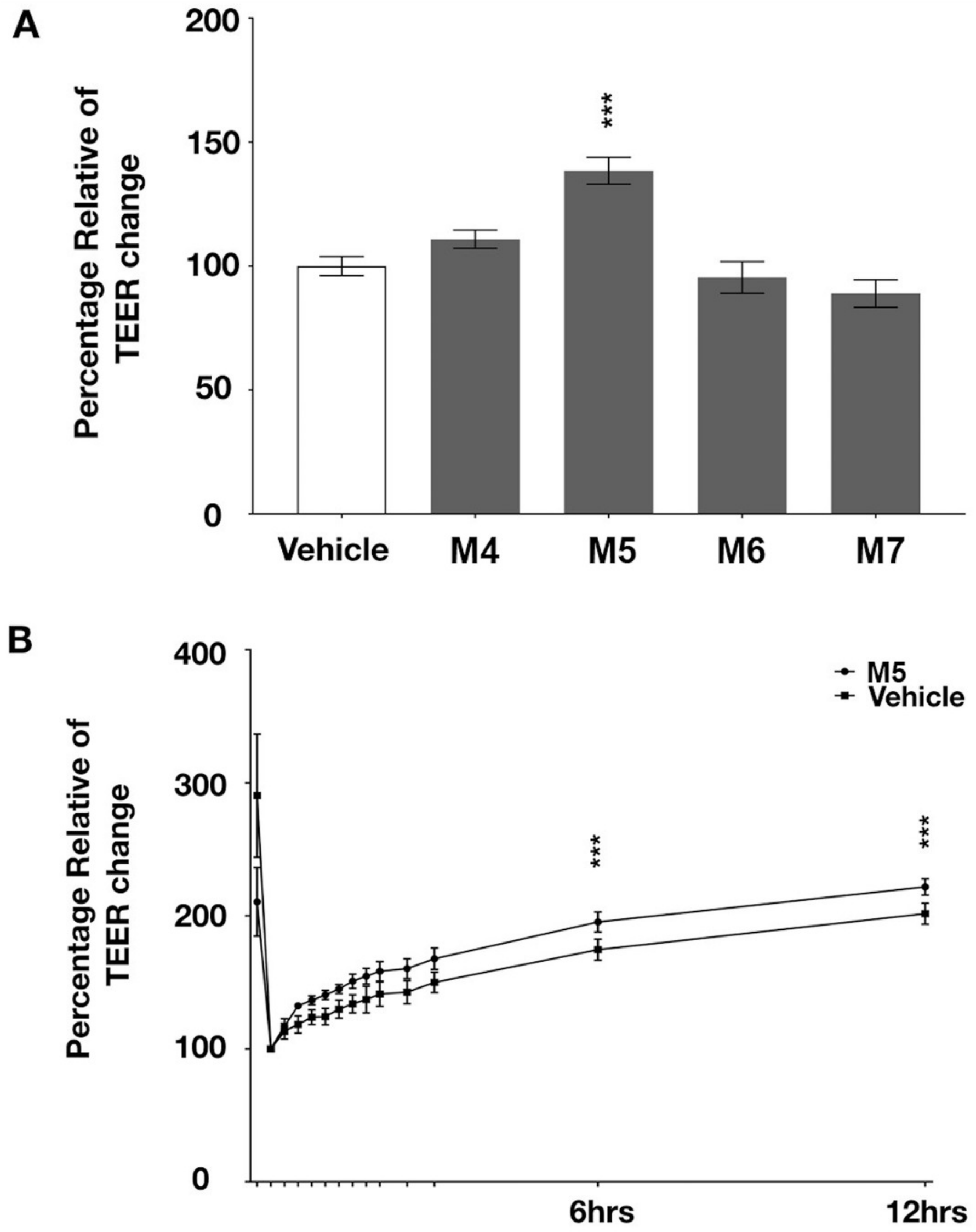

